\title{
Circulating N-terminal brain natriuretic peptide and cardiac function in response to acute systemic hypoxia in healthy humans
}

Ilkka Heinonen ${ }^{1,2,7^{*}}$, Matti Luotolahti ${ }^{4}$, Olli Vuolteenaho ${ }^{6}$, Mikko Nikinmaa ${ }^{5}$, Antti Saraste ${ }^{1,3}$, Jaakko Hartiala ${ }^{4}$, Juha Koskenvuo ${ }^{2,4}$, Juhani Knuuti ${ }^{1}$ and Olli Arjamaa ${ }^{5}$

\begin{abstract}
Background: As it remains unclear whether hypoxia of cardiomyocytes could trigger the release of brain natriuretic peptide (BNP) in humans, we investigated whether breathing normobaric hypoxic gas mixture increases the circulating NT-proBNP in healthy male subjects.

Methods: Ten healthy young men (age $29 \pm 5 \mathrm{yrs}, \mathrm{BMI} 24.7 \pm 2.8 \mathrm{~kg} / \mathrm{m}^{2}$ ) breathed normobaric hypoxic gas mixture $\left(11 \% \mathrm{O}_{2} / 89 \% \mathrm{~N}_{2}\right)$ for one hour. Venous blood samples were obtained immediately before, during, and 2 and 24 hours after hypoxic exposure. Cardiac function and flow velocity profile in the middle left anterior descending coronary artery (LAD) were measured by Doppler echocardiography.

Results: Arterial oxygen saturation decreased steadily from baseline value of $99 \pm 1 \%$ after the initiation hypoxia challenge and reached steady-state level of $73 \pm 6 \%$ within 20-30 minutes. Cardiac output increased from $6.0 \pm 1.2$ to $8.1 \pm 1.6 \mathrm{~L} / \mathrm{min}$ and ejection fraction from $67 \pm 4 \%$ to $75 \pm 6 \%$ (both $\mathrm{p}<0.001$ ). Peak diastolic flow velocity in the LAD increased from $0.16 \pm 0.04$ to $0.28 \pm 0.07 \mathrm{~m} / \mathrm{s}$, while its diameter remained unchanged. In the whole study group, NT-proBNP was similar to baseline $(60 \pm 32 \mathrm{pmol} / \mathrm{ml})$ at all time points. However, at $24 \mathrm{~h}$, concentration of NT-proBNP was higher (34 $\pm 18 \%)$ in five subjects and lower $(17 \pm 17 \%), p=0.002$ between the groups) in five subjects than at baseline.

Conclusion: In conclusion, there is no consistent increase in circulating NT-proBNP in response to breathing severely hypoxic normobaric gas mixture in healthy humans, a possible reason being that the oxygen flux to cardiac myocytes does not decrease because of increased coronary blood flow. However, the divergent individual responses as well as responses in different cardiac diseases warrant further investigations.
\end{abstract}

Keywords: Hypoxia, NT-proBNP, Cardiac function, Humans

\section{Introduction}

Natriuretic peptides are endogenous peptide hormones derived from the myocardium. They have autoregulatory effects to the heart by preventing atrial stretch via regulation of sodium and water balance but they also regulate directly blood vessel tonus and whole body energy homeostasis [1]. Especially brain natriuretic peptide (BNP), which possesses

\footnotetext{
* Correspondence: ilkka.heinonen@utu.fi

${ }^{1}$ Turku PET Centre, University of Turku and Turku University Hospital, PO Box 52, Fl-20521 Turku, Finland

${ }^{2}$ Research Center of Applied and Preventive Cardiovascular Medicine, University of Turku and Turku University Hospital, PO Box 52, Fl-20521 Turku, Finland

Full list of author information is available at the end of the article
}

significant cardioprotective properties, is also frequently measured in patients with cardiovascular diseases as it provides diagnostic and prognostic value in many clinical scenarios. BNP is generally regarded to be released from the ventricles of the heart in response to volume or pressure overload. Emerging evidence from cell culture studies and animal experiments, however, suggests that also hypoxia [2] of cardiomyocytes could trigger the release of BNP $[3,4]$. It has also been shown with cultured human myocardial cells that hypoxia can induce BNP release [5]. Hypoxia effect can be separated from cardiomyocyte stretch [6], although not all human cell culture studies have repeated this response [7]. 
The physiological in vivo relevance of these cell culture findings remains unclear, however, as tissue oxygen levels may never reach such low and long-standing severe hypoxia values as is generally used in vitro studies. Nevertheless, normobaric hypoxic exposure for 60 minutes has been shown to induce slight increase in N-terminal BNP, but this study was performed in highly selected group of subjects that showed either high or low renin-angiotensin system activity [8]. On the other hand, a very severe (corresponding to altitude of $9144 \mathrm{~m}$ ), but short (1-3 min) exposure to hypobaric hypoxia did not cause an increase in BNP levels [9]. Similarly, also a short hypobaric chamber exposure ( $25 \mathrm{~min}$ ) at rest followed by $1 \mathrm{~min}$ of exercise did not lead to immediate increase in circulating BNP in healthy humans [10].

However, it might be that as BNP peptide is stored only in small amounts in secretary granules of ventricular myocytes [11], increase in BNP becomes apparent only after sufficient time period following hypoxia exposure, when gene expression has been triggered by hypoxia to form more of the peptide. Furthermore, cardiac function and coronary blood flow responses have not been monitored simultaneously with hypoxic exposure in these previous human studies, making it difficult to evaluate whether loading conditions for instance are affected and contribute to hypoxia-induced BNP release. Consequently, to elucidate these yet remaining aspects of the possible hypoxia-induced BNP release in humans in vivo, we measured circulating $\mathrm{N}$-terminal proBNP levels repeatedly during breathing severely hypoxic gas mixture, and 2 hours and 24 hours after the exposure. Furthermore, cardiac function was also assessed comprehensively and simultaneously during hypoxic exposure, to further understand the possible independent role of hypoxia to trigger BNP release from cardiomyocytes.

\section{Methods \\ Subjects}

Ten healthy Caucasian young men (age $29 \pm 5$ yrs, height $181 \pm 4 \mathrm{~cm}$, weight $81 \pm 11 \mathrm{~kg}$, BMI $24.7 \pm 2.8 \mathrm{~kg} / \mathrm{m}^{2}$ ) volunteered to participate in this study. The purpose, nature, and potential risks of the study were explained to the subjects before they gave their written informed consent to participate. The subjects were normotensive nonsmokers with no history of hypercholesterolemia, not taking any medications and had never experienced angina symptoms. The normal health status of the subjects was confirmed by a medical doctor by clinical examination, ECG and cardiac echocardiography before the experiments. The study was performed according to the Declaration of Helsinki and was approved by the Ethics Committee of the Hospital District of South-West Finland.

\section{Hypoxic exposure and blood sampling}

The subjects were requested to avoid strenuous physical exercise in the $48 \mathrm{~h}$ prior to the experiments. The hypoxic exposure was performed in a fasting state and always started around 9 AM with study preparations. In these preparations 12-lead ECG was positioned and an antecubital vein was cannulated for blood sampling. Venous blood samples were obtained before, and 2 and 24 hours after hypoxic exposure and analyzed by standard hospital practices. After initial preparations, subjects breathed normobaric hypoxic gas mixture $\left(11 \% \mathrm{O}_{2} / 89 \% \mathrm{~N}_{2}\right)$ for one hour. Arterial oxygen saturation was continuously measured by pulse oximetry and valid plethysmogram signal was confirmed throughout the studies. All of these measurements were performed when subjects were resting supine.

\section{Analysis of BNP}

Plasma samples $(0.9 \mathrm{ml})$ were first concentrated by extraction with SepPak C18 cartidges, to enable the quantification of the very low levels of circulating BNP peptides in healthy young subjects. NT-proBNP concentrations were determined by immunoassay specific to human NT-proBNP1029 as previously reported $[12,13]$. NT-proBNP is a product of the same precursor and therefore reflects the secretion of the biologically active peptide BNP.

\section{Echocardiography}

Transthoracic 2 dimensional (2D) and Doppler echocardiographic studies were performed with Acuson Sequoia 512 (Siemens Medical Solutions, USA) equipped with 3V2c transducer at rest and $20 \mathrm{~min}$ after beginning of the hypoxia. The digitized images were stored in Syngo Dynamics-system (Siemens, Siemens Medical Solutions, USA) for later analysis. The study subjects were examined in a left lateral decubitus position. Left and right ventricular dimensions and left ventricular wall thickness and ejection fraction and left atrial dimension were measured in parasternal long axis view from 2-dimensionally guided $M$ mode tracings using second harmonic $4.25 \mathrm{MHz}$ scanning frequency. Pulmonary artery flow velocity (pulsed wave Doppler) was measured in the parasternal short axis view at the level of aortic root. The mitral inflow velocities and blood flow velocities in pulmonary veins (pulsed wave Doppler), pressure gradient between right ventricle and right atria (continuous wave Doppler), tissue Doppler velocities of the mitral annulus and M-mode traced valvular ring amplitudes were all recorded from apical 4-Chamber view. Vena cava inferior was examined from the subcostal view. The mean of at least three consecutive cardiac cycles was always calculated and averaged.

Diameter and blood flow velocity (pulsed wave Doppler) were measured in the mid left anterior descending coronary artery using a modified parasternal short axis view focusing on the interventricular sulcus 
using 15 L8 linear transducer as described earlier $[14,15]$.

\section{Statistical analysis}

Statistical analysis was performed using SAS/STAT statistical software (version 9.2, SAS institute Inc., Cary, NC, USA). Two-tailed Student's t-test or one-way (or twoway in terms of group differences) ANOVA for repeated measurements was used for the analysis of statistical differences when appropriate. ANOVA was followed by Tukey post-hoc test if necessary to detect differences in different study time points, or groups. $\mathrm{P}$ values $<0.05$ were considered statistically significant. All data are shown as mean $\pm \mathrm{SD}$.

\section{Results}

All of the subjects had normal baseline arterial oxygen saturation of $99 \pm 1 \%$ at baseline, which decreased steadily after the initiation of hypoxic breathing and reached a stable steady-state level $(73 \pm 6 \%)$ within $20-30$ min after the initiation of hypoxic exposure based on continuous pulse oximetry (Figure 1A). This level of oxygen saturation was maintained until the removal of the hypoxic mask after 1 hour, after which saturation reached normal, pre-hypoxic values $(99 \pm 1 \%$ ) within 1 min (Figure 1A). Hemoglobin concentration was increased towards the end of the hypoxic exposure, but returned to pre-hypoxia levels after 2 hours of returning to normoxic conditions (Figure 1B).

Heart rate response followed a similar, but reversed pattern to that of arterial oxygen saturation, being $58 \pm$ $9 \mathrm{bpm}$ at resting baseline, $79 \pm 11$ during hypoxia, and $61 \pm 1 \mathrm{bpm}$ two hours after the end of hypoxic exposure (Figure 2A). Systolic blood pressure did not change (Figure $2 \mathrm{~B}$ ) in response to hypoxia ( $\mathrm{p}=0.42$ in ANOVA), but diastolic blood pressure was slightly lower in the end of hypoxia than at baseline or during normoxic recovery from the hypoxia exposure (Figure 2C).

In Table 1 parameters describing cardiac function at baseline and in hypoxia are presented. No regional wall motion abnormalities were detected. Of note, hypoxia led to increased cardiac output and ejection fraction, but atrial sizes did not change. Left coronary artery blood

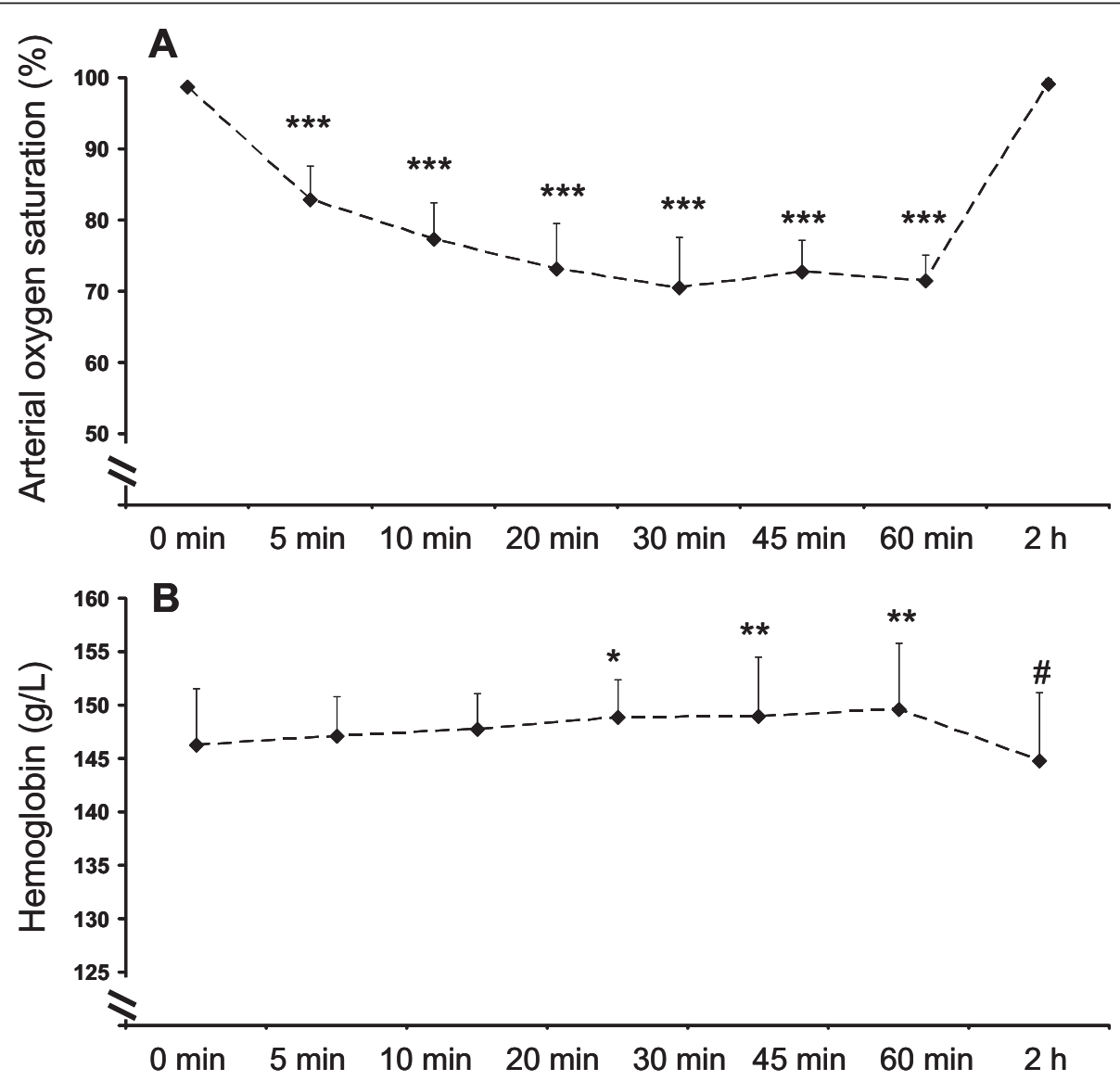

Figure 1 Arterial oxygen saturation, as determined by pulse oxymetry (A), and venous haemoglobin concentration (B) before hypoxia $(0 \mathrm{~min})$, repeatedly during $60 \mathrm{~min}$ of hypoxic exposure, and 2 hours after the exposure. ${ }^{*} p<0.05,{ }^{* *} p<0.01$, and ${ }^{* * *} p<0.001$ compared to 0 min and $2 \mathrm{~h}, \# \mathrm{p}<0.05$ compared to $60 \mathrm{~min}$. 


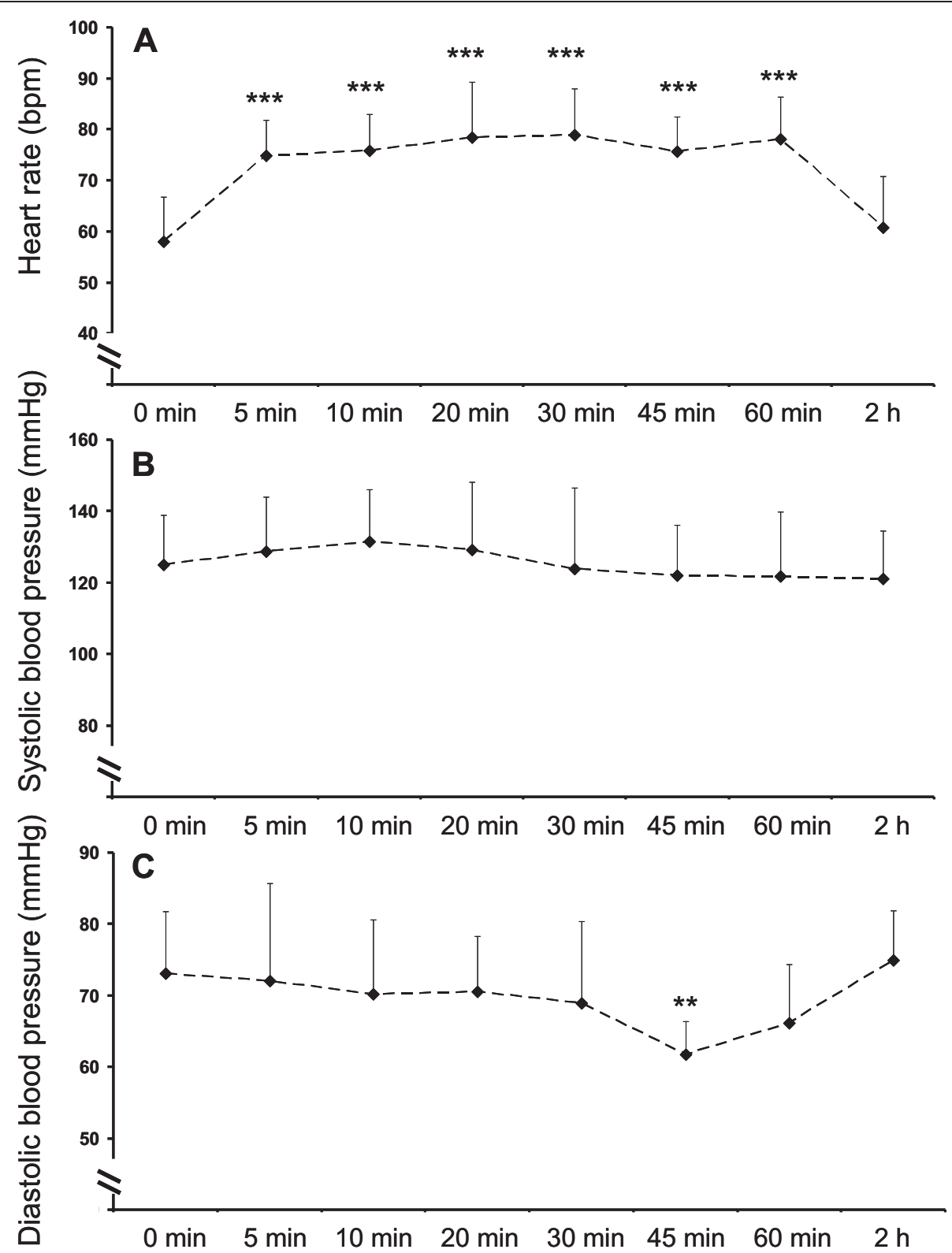

Figure 2 Heart rate (A), and systolic (B) and diastolic blood pressures (C) before hypoxia ( 0 min), repeatedly during 60 min of hypoxic exposure, and 2 hours after the exposure. ${ }^{* *} p<0.01$, and ${ }^{* *} p<0.001$ compared to 0 min and $2 \mathrm{~h}$.

velocity was also increased, but its diameter remained unchanged in response to hypoxia. A decrease in E/Aratio was also observed.

At the whole study group level there was no change in NT-proBNP concentration in response to applied systemic hypoxia (Figure 3A). However, two distinct NTproBNP response groups to hypoxia were also identified, as in five subjects the $24 \mathrm{~h}$ concentration of NT-proBNP was elevated on average $34 \pm 18 \%$ as compared with the baseline concentration, while in five subjects it was reduced by an average of $17 \pm 17 \%$ lower (Figure 3B). NTproBNP either increased or decreased in all of these five subjects. Mean heart rate was consistently higher throughout the experiment in the five subjects with elevated $B N P$ at 24 hours $(62 \pm 11,77 \pm 8,78 \pm 9,81 \pm 10$, $84 \pm 7,78 \pm 8,81 \pm 6,65 \pm 11 \mathrm{bpm}$ at baseline before hypoxia, and $5,10,20,30,45,60$ min time points during, and 2 hours after hypoxia, respectively) as compared to the other five individuals $(55 \pm 5,73 \pm 6,74 \pm 5,76 \pm$ $12,73 \pm 9,73 \pm 5,76 \pm 11,56 \pm 6$, respectively, $\mathrm{p}<0.01$ for the group difference in two-way ANOVA, hypoxia"group interaction $\mathrm{p}=\mathrm{NS}$ ), but there were no group differences, or even trends towards differences, in arterial oxygen saturation, haemoglobin concentration, blood 
Table 1 Cardiac function at baseline and after 20 minutes exposure to hypoxic stimulus

\begin{tabular}{lll}
\hline & Baseline & Hypoxia \\
\hline Cardiac output (L/min) & $6.0 \pm 1.2$ & $8.1 \pm 1.6^{* * *}$ \\
Ejection fraction (\%) & $67 \pm 4$ & $75 \pm 6^{* * *}$ \\
RV ED diameter (mm) & $25 \pm 4$ & $26 \pm 3$ \\
RA diameter (mm) & $20 \pm 4$ & $19 \pm 2$ \\
LA diameter (mm) & $39 \pm 4$ & $38 \pm 3$ \\
PA blood flow velocity (m/s) & $0.8 \pm 0.1$ & $1.0 \pm 0.1^{*}$ \\
Vena cava inferior (mm) & $16.8 \pm 4.6$ & $15.2 \pm 4.2$ \\
RV-to-RA pressure gradient (mmHg) & $28 \pm 5$ & $31 \pm 3^{*}$ \\
RV E'/ A' (cm/s) & $1.5 \pm 0.4$ & $1.4 \pm 0.2$ \\
RV S $(\mathrm{cm} / \mathrm{s})$ & $0.20 \pm 0.04$ & $0.24 \pm 0.04^{*}$ \\
Pulmonary vein s/d ratio & $1.3 \pm 0.2$ & $1.4 \pm 0.3$ \\
TAPSE (cm) & $2.6 \pm 0.2$ & $3.0 \pm 0.3^{* *}$ \\
LAD diameter (cm) & $5.62 \pm 0.64$ & $5.60 \pm 0.64$ \\
LAD blood flow velocity (m/s) & $0.16 \pm 0.4$ & $0.28 \pm 0.07^{* *}$ \\
LV lateral wall E'/A' & $1.9 \pm 0.7$ & $1.5 \pm 0.6$ \\
LV septal wall E'/A' & $1.6 \pm 0.3$ & $1.5 \pm 0.3$ \\
LV septum Sm (cm/s) & $0.12 \pm 0.02$ & $0.15 \pm 0.05$ \\
LV lateral wall Sm (cm/s) & $0.16 \pm 0.06$ & $0.19 \pm 0.06$ \\
Lateral mitral annular displacement (cm) & $1.5 \pm 0.2$ & $1.8 \pm 0.2^{* *}$ \\
Septal mitral annular displacement (cm) & $1.5 \pm 0.1$ & $1.7 \pm 0.3^{*}$ \\
E/A -ratio & $1.6 \pm 0.3$ & $1.4 \pm 0.2^{*}$ \\
\hline LV I & &
\end{tabular}

$\mathrm{LV}=$ left ventricle, $\mathrm{RV}=$ Right ventricle, $\mathrm{LA}=$ left atrium, $\mathrm{RA}=$ right atrium, TAPSE = Tricuspid annular plane systolic excursion, $\mathrm{PA}=$ pulmonary artery $E^{\prime}=$ tissue-Doppler early diastolic velocity, $A^{\prime}=$ tissue-Doppler late diastolic velocity, $\mathrm{S}_{\mathrm{m}}=$ tissue-Doppler systolic velocity, $\mathrm{E}=$ Mitral inflow early peak velocity, $A=$ Mitral inflow atrial peak velocity. ${ }^{*} p<0.05,{ }^{* *} p<0.01,{ }^{* * *} p<0.001$ compared to baseline. $\mathrm{LAD}=$ the left anterior descending coronary artery.

pressures or any of the echocardiography variables measured in the present study (data not shown).

\section{Discussion}

In the present study we measured cardiac function, coronary blood flow velocity and peripheral circulating levels of NT-proBNP in response to breathing severely hypoxic gas mixture in healthy young male volunteers to study the possibility that hypoxia per se could trigger the release of NT-proBNP. We did not find any consistent increase in circulating NT-proBNP, which was likely due to the fact that despite the marked reduction of oxygen saturation, oxygen supply to the cardiomyocytes was fully compensated for by the increased coronary blood flow in these healthy young adults. Nevertheless, two distinct NT-proBNP response groups to hypoxia were also identified in the present study. It will be discussed that this divergent response is likely related to hypoxic regulation of BNP genetic responses, and in the long term might determine individual adaptation to chronic hypoxia.
Our findings in terms of cardiac responses to acute systemic hypoxia are well in accordance with the established literature, which also describes tachycardia and largely unchanged blood pressure as one of the key feature of hypoxic physiological response [16-18]. Although hypoxia suppresses myocardial contractility in some studies that have been performed in vitro, also our finding of higher ejection fraction, thus improved emptying of the ventricle, supports the notions that hypoxia may induce compensatory improvement in cardiac pump function in vivo in healthy humans $[16,19]$. Moreover, although a decrease in early-tolate filling (E/A-ratio) of the ventricle was also observed in the present study, which would normally suggest impaired diastolic filling, it has been previously documented that this is largely the result of decreased early filling due to preload reduction, while there is simultaneously a greater contribution of atrial contraction in acute systemic hypoxia [16].

However, when it comes to the NT-proBNP levels during acute hypoxic exposure, as sampled repeatedly during the exposure, as well as the 2 and $24 \mathrm{~h}$ level after hypoxic exposure, we observed that it remained essentially similar to the pre-exposure baseline NT-proBNP level. This was the case even when the arterial oxygen saturation dropped close to $70 \%$ during the severe acute hypoxic exposure. However, despite this reduction it is likely that absolute oxygen supply to heart was not actually decreased, as coronary blood flow velocity increased by $75 \%$ while the diameter of left anterior coronary artery remained unchanged. Therefore, it is apparent that the investigation of BNP release is hampered by the fact that despite the reduction of oxygen saturation, oxygen supply of the cardiomyocytes is mostly fully compensated by the increased coronary blood flow in healthy young adults.

From the present results it is clear that breathing severely hypoxic gas mixture does not elicit increased NT-proBNP level, as despite the decreased arterial oxygen saturation cardiac myocytes can obtain unchanged (or even increased) amount of oxygen as myocardial blood flow increases markedly [20]. Myocardial hypoxia may however occur if coronary blood flow cannot respond to reduced oxygen saturation or oxygen demand is excessively increased, as might happen in many cardiac patients in which BNP is frequently determined as a diagnostic measure. Local cardiac hypoxia typical for many cardiac patients may cause the increase in circulating NT-proBNP levels, in addition to wellestablished causes of pressure and volume overload [21-23], but unfortunately, ischemia-prone individuals were not investigated in the present study and this aspect warrants further experimentation. Another possible explanation in addition to the blood flow compensation is that our onehour hypoxic exposure was too short to induce the expression of BNP gene. In long-term experiments circulating BNP has been shown to be increased especially in subjects who suffer from chronic mountain sickness [24-26], but 

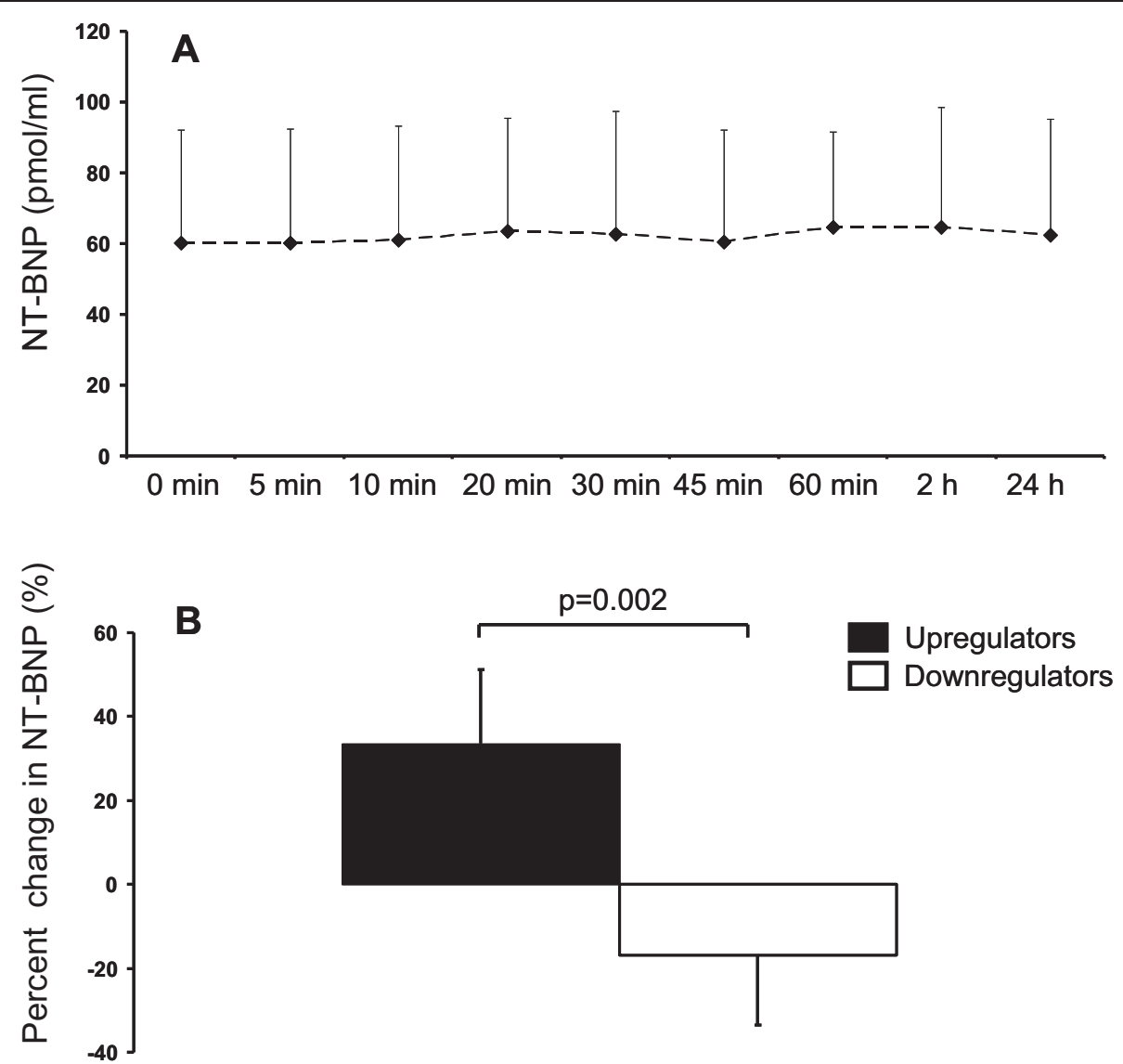

Figure 3 NT-proBNP responses. Circulating NT-proBNP concentration (A) before hypoxia ( 0 min), repeatedly during 60 min of hypoxic exposure, and 2 and 24 hours after the exposure. In panel $\mathbf{B}$ mean per cent change in NT-proBNP of two divergent groups ( $\mathrm{n}=5$ in both groups) are presented, which showed either increase ("upregulators") or reduction ("downregulators") from baseline to 24 hours of hypoxic exposure in NT-proBNP concentration.

clear interpretation if hypoxia per se triggers BNP release remains open, as these studies are also confounded by several other stress factors, such as physical stress caused by hiking to and on the mountains and not be hypoxia per se. These findings have neither been confirmed by all studies [27]. Preoperative BNP levels are also within normal levels in patients with tetralogy of Fallot (severe congenital cyanotic heart defect) who have generally at least moderate decreases in systemic oxygen saturation [28].

Finally, despite the fact that no increase in mean levels of NT-proBNP was detected in the present study, we observed two differently responding groups; in five subjects, the $24 \mathrm{~h}$ concentration of NT-proBNP was higher than the baseline concentration, while in five subjects it was lower. As these divergent responses could not be related to changes in cardiac function during hypoxia, it is possible that these differing responses are due to differences in single nucleotide polymorphisms in gene areas regulating BNP expression. In this regard it is interesting that BNP gene is under the direct control of HIF (hypoxia-inducible factor) [5,7,29], which is the master regulator of hypoxic responses. Thus, it remains to be investigated whether these two divergent BNP responses could be localized to differences in single nucleotide polymorphisms that are under the control of HIF.

Moreover, it might also be that these differences in NTBNP responses also determine the susceptibility to hypoxic mountain sickness [24-26]. In this regard, circulating BNP has been shown by Ge and Mo et al. to be predictive of chronic mountain sickness [26]. However, in their study patients with mountain sickness also had higher pulmonary artery pressures and lower arterial oxygen saturations compared to subjects who did not get altitude sickness [26]. Therefore, it might be that also in this case the increase in BNP is just a result of increased cardiac pressures [21-23], but further studies are still warranted to investigate the emerging evidence from animal studies that hypoxia, and/ or ischemia, per se could trigger the release of brain natriuretic peptides also in humans.

\section{Methodological considerations}

It is a limitation that we did not measure NT-proBNP levels directly from coronary sinus, but instead peripheral venous 
site was used for sampling. Together with its binding to receptors, this may have caused NT-proBNP to be diluted to body fluids and lowered the detection limit to observe changes. However, not even a trend towards changes was observed as whole study group level, although on the other hand changes were robust when investigated at the subgroup level indicating the validity of peripheral sampling. Moreover, circulating levels of BNP show a close relationship with its content in myocardium [30], making its measurement from peripheral blood valid approach, especially in healthy volunteers who have no medical or other reasons for cardiac catheterization.

In conclusion, we did not find increase in circulating NT-BNP in response to breathing severely hypoxic gas mixture in humans in the present study. However, cardiac myocytes did not probably experience reduced amount of oxygen, as despite the fact that arterial oxygen saturation was markedly reduced, oxygen supply to the cardiomyocytes was likely fully compensated for by the increased coronary blood flow in the studied healthy young adults. Acute studies patients with cardiac diseases, especially those with limited possibility to adjust coronary blood flow, and chronic hypoxic exposure studies in healthy humans under well-controlled conditions are required to conclusively show the role of hypoxia in triggering NTBNP release.

\section{Competing interests}

The author declares that they have no competing interests.

\section{Authors' contributions}

All authors have participated in study planning, performing and writing the manuscript. All have read and approved submission of the final version of the manuscript.

\section{Acknowledgements}

The study was conducted within the Centre of Excellence in Molecular Imaging in Cardiovascular and Metabolic Research - supported by the Academy of Finland, University of Turku, Turku University Hospital and Åbo Akademi University. The authors want to thank the personnel of Turku PET Centre for their excellent assistance. The study was financially supported by the Academy of Finland (Grants 251572 and 258078, and Centre of Excellence funding), and the Hospital District of Southwest Finland.

\footnotetext{
Author details

${ }^{1}$ Turku PET Centre, University of Turku and Turku University Hospital, PO Box 52, Fl-20521 Turku, Finland. ${ }^{2}$ Research Center of Applied and Preventive Cardiovascular Medicine, University of Turku and Turku University Hospital, PO Box 52, Fl-20521 Turku, Finland. ${ }^{3}$ Heart Center, University of Turku and Turku University Hospital, PO Box 52, FI-20521 Turku, Finland. ${ }^{4}$ Department of Clinical Physiology and Nuclear Medicine, University of Turku and Turku University Hospital, PO Box 52, Fl-20521 Turku, Finland. ${ }^{5}$ Department of Biology, University of Turku and Turku University Hospital, PO Box 52, $\mathrm{Fl}-20521$ Turku, Finland. Institute of Biomedicine, University of Oulu, Oulu, Finland. 'Division of Experimental Cardiology, Thoraxcenter, Erasmus MC, University Medical Center Rotterdam, Rotterdam, The Netherlands.
}

Received: 17 February 2014 Accepted: 9 June 2014

Published: 3 July 2014

\section{References}

1. Moro C, Lafontan M: Natriuretic peptides and cGMP signaling control of energy homeostasis. Am J Physiol Heart Circ Physiol 2013, 304:H358-H368.

2. Nikinmaa M: "What is hypoxia?" Acta Physiol (Oxf) 2013, doi:10.1111/apha.12146.

3. Arjamaa $\mathrm{O}$, Nikinmaa M: Hypoxia regulates the natriuretic peptide system. Int J Physiol Pathophysiol Pharmacol 2011, 3:191-201.

4. Arjamaa O, Nikinmaa M: Natriuretic peptides in hormonal regulation of hypoxia responses. Am J Physiol Regul Integr Comp Physiol 2009, 296:R257-R264.

5. Casals G, Ros J, Sionis A, Davidson MM, Morales-Ruiz M, Jimenez W: Hypoxia induces B-type natriuretic peptide release in cell lines derived from human cardiomyocytes. Am J Physiol Heart Circ Physiol 2009, 297:H550-H555.

6. Mollmann H, Nef HM, Kostin S, Dragu A, Maack C, Weber M, Troidl C, Rolf A, Elsasser A, Bohm M, Brantner R, Hamm CW, Holubarsch CJ: Ischemia triggers BNP expression in the human myocardium independent from mechanical stress. Int J Cardiol 2010, 143:289-297.

7. Luo Y, Jiang C, Belanger AJ, Akita GY, Wadsworth SC, Gregory RJ, Vincent KA: A constitutively active hypoxia-inducible factor-1alpha/VP16 hybrid factor activates expression of the human B-type natriuretic peptide gene. Mol Pharmacol 2006, 69:1953-1962.

8. Due-Andersen R, Pedersen-Bjergaard U, Hoi-Hansen T, Olsen NV, Kistorp C, Faber J, Boomsma F, Thorsteinsson B: NT-pro-BNP during hypoglycemia and hypoxemia in normal subjects: impact of renin-angiotensin system activity. J Appl Physiol 2008, 104:1080-1085.

9. Karadag $R$, Sen A, Yildirim N, Basmak H, Golemez H, Cakir E, Akin A: The relation between intraocular pressure change and plasma natriuretic peptide under simulated hypobaric conditions. Indian $J$ Ophthalmol 2010, 58:195-198.

10. Woods D, Hooper T, Mellor A, Hodkinson P, Wakeford R, Peaston B, Ball S, Green N: Brain natriuretic peptide and acute hypobaric hypoxia in humans. J Physio/ Sci 2011, 61:217-220.

11. Levin ER, Gardner DG, Samson WK: Natriuretic peptides. N Engl J Med 1998, 339:321-328.

12. Ala-Kopsala M, Magga J, Peuhkurinen K, Leipala J, Ruskoaho $H_{\text {, }}$ Leppaluoto J, Vuolteenaho O: Molecular heterogeneity has a major impact on the measurement of circulating $\mathrm{N}$-terminal fragments of A- and B-type natriuretic peptides. Clin Chem 2004, 50:1576-1588.

13. Ala-Kopsala M, Moilanen AM, Rysa J, Ruskoaho H, Vuolteenaho O: Characterization of molecular forms of $\mathrm{N}$-terminal B-type natriuretic peptide in vitro. Clin Chem 2010, 56:1822-1829.

14. Kiviniemi TO, Saraste M, Koskenvuo JW, Airaksinen KE, Toikka JO, Saraste A, Parkka JP, Hartiala JJ: Coronary artery diameter can be assessed reliably with transthoracic echocardiography. Am J Physiol Heart Circ Physiol 2004, 286:H1515-H1520.

15. Kiviniemi TO, Toikka JO, Koskenvuo JW, Saraste A, Saraste M, Parkka JP, Raitakari OT, Hartiala JJ: Vasodilation of epicardial coronary artery can be measured with transthoracic echocardiography. Ultrasound Med Biol 2007, 33:362-370.

16. Naeije R: Physiological adaptation of the cardiovascular system to high altitude. Prog Cardiovasc Dis 2010, 52:456-466

17. Hultgren HN, Grover RF: Circulatory adaptation to high altitude. Annu Rev Med 1968, 19:119-152.

18. Bartsch P, Gibbs JS: Effect of altitude on the heart and the lungs. Circulation 2007, 116:2191-2202.

19. Dedobbeleer C, Hadefi A, Naeije R, Unger P: Left ventricular adaptation to acute hypoxia: a speckle-tracking echocardiography study. J Am Soc Echocardiogr 2013, 26(7):736-745.

20. Namdar M, Koepfli P, Grathwohl R, Siegrist PT, Klainguti M, Schepis T, Delaloye R, Wyss CA, Fleischmann SP, Gaemperli O, Kaufmann PA: Caffeine decreases exercise-induced myocardial flow reserve. J Am Coll Cardiol 2006, 47:405-410.

21. Tenhunen O, Szokodi I, Ruskoaho H: Posttranscriptional activation of BNP gene expression in response to increased left ventricular wall stress: role of calcineurin and PKC. Regul Pept 2005, 128:187-196.

22. Maeder MT, Mariani JA, Kaye DM: Hemodynamic determinants of myocardial B-type natriuretic peptide release: relative contributions of systolic and diastolic wall stress. Hypertension 2010, 56:682-689.

23. Wiese S, Breyer T, Dragu A, Wakili R, Burkard T, Schmidt-Schweda S, Fuchtbauer EM, Dohrmann U, Beyersdorf F, Radicke D, Holubarsch CJ: Gene expression of brain natriuretic peptide in isolated atrial and ventricular 
human myocardium: influence of angiotensin II and diastolic fiber length. Circulation 2000, 102:3074-3079.

24. Woods D, Hooper T, Hodkinson P, Ball S, Wakeford R, Peaston B, Bairsto C, Green N, Mellor A: Effects of altitude exposure on brain natriuretic peptide in humans. Eur J Appl Physiol 2011, 111:2687-2693.

25. Woods DR, Begley J, Stacey M, Smith C, Boos CJ, Hooper T, Hawkins A, Hodkinson P, Green N, Mellor A: Severe acute mountain sickness, brain natriuretic peptide and NT-proBNP in humans. Acta Physiol (Oxf) 2012, 205:349-355.

26. Ge RL, Mo VY, Januzzi JL, Jin G, Yang Y, Han S, Wood MJ, Levine BD: B-type natriuretic peptide, vascular endothelial growth factor, endothelin-1, and nitric oxide synthase in chronic mountain sickness. Am J Physiol Heart Circ Physiol 2011, 300:H1427-H1433.

27. Toshner MR, Thompson AA, Irving JB, Baillie JK, Morton JJ, Peacock AJ: NT-proBNP does not rise on acute ascent to high altitude. High Alt Med Biol 2008, 9:307-310.

28. Koch A, Zink S, Singer H: B-type natriuretic peptide in paediatric patients with congenital heart disease. Eur Heart J 2006, 27:861-866.

29. Weidemann A, Klanke B, Wagner M, Volk T, Willam C, Wiesener MS, Eckardt $\mathrm{KU}$, Warnecke C: Hypoxia, via stabilization of the hypoxia-inducible factor HIF-1alpha, is a direct and sufficient stimulus for brain-type natriuretic peptide induction. Biochem J 2008, 409:233-242.

30. Goetze JP, Christoffersen C, Perko M, Arendrup H, Rehfeld JF, Kastrup J, Nielsen LB: Increased cardiac BNP expression associated with myocardial ischemia. FASEB J 2003, 17:1105-1107.

doi:10.1186/1479-5876-12-189

Cite this article as: Heinonen et al:: Circulating $\mathrm{N}$-terminal brain

natriuretic peptide and cardiac function in response to acute systemic hypoxia in healthy humans. Journal of Translational Medicine 2014 12:189.

\section{Submit your next manuscript to BioMed Central and take full advantage of:}

- Convenient online submission

- Thorough peer review

- No space constraints or color figure charges

- Immediate publication on acceptance

- Inclusion in PubMed, CAS, Scopus and Google Scholar

- Research which is freely available for redistribution 